\title{
EVALUATION OF NUTRITIONAL PRACTICES IN A TUNISIAN NEONATAL INTENSIVE CARE UNIT
}

\section{Imene Ayadi Dahmane, Emira Ben Hamida, Mouadh Bou Ali, Bassem \\ Rabaii, Zahra Marrakchi \\ Department of Neonatology. Charles Nicolle Hospital. Tunis. Tunisia.}

Introduction: Postnatal growth restriction is a universal problem in very premature infants. Nutritional practices leading to proteino-caloric deficit may participate to its occurrence. The aims of the study were to describe nutritional practices in premature infants less than 32 weeks and less than $1500 \mathrm{~g}$ in a Tunisian neonatal intensive care unit.

\section{Methods :}

We conducted a retrospective study in neonatal resuscitation unit of Charles Nicolle Hospital in Tunis, over a period of three years (January 2011-December 2013).

We included all the premature infants of gestational age $<32 S A$ and $P N<1500$ g who survived the discharge from the hospital.

59 preterm infants were eligible.

General characteristics of the population

Gestation al age

Birth Weigh (g)

$1250 \mathrm{~g} \pm 170$

(850-1500)

Respiratory distress

49.2

syndrome (\%)

Apnea (\%)

28.8

Sepsis (\%)

17

Hemodynamic

30.5

disorders (\%)

Necrotizing

3.4

enterocolitis (\%)

\begin{tabular}{lcc|}
\hline \multicolumn{3}{c|}{ Enteral nutritional practices } \\
\hline Date & $\begin{array}{c}\text { Enteral nutrition } \\
>50 \%\end{array}$ & $\begin{array}{c}\text { Totale enteral } \\
\text { nutrition }\end{array}$ \\
$<$ day 7 & $24,1 \%$ & $3.4 \%$ \\
$7-14$ days & $46,6 \%$ & $45.8 \%$ \\
$15-21$ days & $20,7 \%$ & $32.2 \%$ \\
$>21$ days & $8,6 \%$ & $6.8 \%$
\end{tabular}

\begin{tabular}{|c|c|c|}
\hline \multicolumn{3}{|c|}{ Parenteral nutritional practices } \\
\hline $\begin{array}{l}\text { Introduction of } \\
\text { proteins }\end{array}$ & day 1 & $100 \%$ \\
\hline Initiation dose & $\begin{array}{l}1 \mathrm{~g} / \mathrm{kg} / \mathrm{d} \\
1.5 \mathrm{~g} / \mathrm{kg} / \mathrm{d}\end{array}$ & $\begin{array}{l}85 \% \\
15 \%\end{array}$ \\
\hline Progression dose & $\begin{array}{l}0.5 \mathrm{~g} / \mathrm{kg} / \mathrm{d} \\
1 \mathrm{~g} / \mathrm{kg} / \mathrm{d}\end{array}$ & $\begin{array}{l}10 \% \\
90 \%\end{array}$ \\
\hline Maximum intake & $\begin{array}{l}3.5 \mathrm{~g} / \mathrm{kg} / \mathrm{d} \\
4 \mathrm{~g} / \mathrm{kg} / \mathrm{d}\end{array}$ & $\begin{array}{l}15 \% \\
85 \%\end{array}$ \\
\hline $\begin{array}{l}\text { Introduction of } \\
\text { lipids }\end{array}$ & \begin{tabular}{|l} 
day1 \\
day2 \\
day3 \\
$\geq$ day4
\end{tabular} & $\begin{array}{l}27 \% \\
52 \% \\
13 \% \\
8 \%\end{array}$ \\
\hline Initiation dose & $1 \mathrm{~g} / \mathrm{kg} / \mathrm{d}$ & $100 \%$ \\
\hline Maximum intake & $3.5 \mathrm{~g} / \mathrm{kg} / \mathrm{d}$ & $100 \%$ \\
\hline $\begin{array}{l}\text { Introduction of } \\
\text { carbohydrates }\end{array}$ & day1 & $100 \%$ \\
\hline Initiation dose & $\begin{array}{l}8 \mathrm{~g} / \mathrm{kg} / \mathrm{d} \\
8-9 \mathrm{~g} / \mathrm{kg} / \mathrm{d} \\
9-12 \mathrm{~g} / \mathrm{kg} / \mathrm{d}\end{array}$ & $\begin{array}{c}80 \% \\
15 \% \\
5 \%\end{array}$ \\
\hline Maximum intake & $\begin{array}{l}15-16 \mathrm{~g} / \mathrm{kg} / \mathrm{d} \\
16-17 \mathrm{~g} / \mathrm{kg} / \mathrm{d} \\
18 \mathrm{~g} / \mathrm{kg} / \mathrm{d}\end{array}$ & $\begin{array}{l}5 \% \\
20 \% \\
75 \%\end{array}$ \\
\hline
\end{tabular}

Conclusion: Our results show that the nutritional practices adopted in our NICU are consistent with the current nutrition recommendations for premature infants. 